\title{
Association of MHC antigens with susceptibility to and severity of rheumatoid arthritis in multicase families
}

\author{
D J WALKER, ${ }^{1}$ M GRIFFITHS ${ }^{1}$ P DEWAR, ${ }^{*}$ E COATES ${ }^{2}$ W C DICK, \\ M THOMPSON,${ }^{1}$ AND I D GRIFFITHS ${ }^{1}$
}

From the ${ }^{1}$ Department of Rheumatology, Newcastle upon Tyne, and the ${ }^{2}$ Regional Blood Transfusion Service, Newcastle upon Tyne

SUMmARY A study of HLA association with rheumatoid arthritis (RA) in multicase families has been performed in north east England. Two hundred and nineteen individuals from 13 families were assessed for the presence of RA, and all were HLA typed. Thirty nine were found to have classical or definite RA by American Rheumatism Association (ARA) criteria. Thirty-five (90\%) of these possess HLA-DR4, confirming the previously reported association of RA with DR4. A further 19 individuals were found to have probable RA or gave a convincing history of previous inflammatory polyarthritis. Thirteen $(68 \%)$ of these possess HLA-DR4, and this is not significantly different from non-affected family members of whom $63 \%$ possess DR 4 . These results suggest that HLA-DR4 is associated only with the more severe forms of RA.

Homozygosity for HLA-DR4 was not associated with either earlier onset or more severe disease when compared with heterozygous DR4. Possession of the haplotype most commonly inherited with the RA in individual families was not associated with earlier onset but may be associated with more severe disease. The severity of RA appears to be influenced by the major histocompatibility complex (MHC) in these families.

Key words: inflammatory polyarthritis, HLA, haplotype, $x$-ray grading.

Both genetic and environmental factors have an important role in the aetiology of RA. Family clustering has been reported; the estimated population prevalence of $1-2 \%$ rises to $3-8 \%$ in first degree relatives of RA sufferers and $33 \%$ concordance in monozygotic twins. ${ }^{1}$ An association between RA and the MHC is now well established. RA as seen in hospitals is clearly associated with both HLA-Dw4 and HLA-DR4. ${ }^{2-4}$ Association has also been shown with complement $\mathrm{C} 4$ isotype, probably because of linkage disequilibrium with DR4. ${ }^{5}$ However, association has been shown with properdin factor, $\mathrm{BfS}$, and this was not the result of linkage disequilibrium with DR4. ${ }^{6}$ This finding suggests that sixth chromosome genes other than those coding for HLA-DR4 and Dw4 may be of importance in the aetiopathogenesis of RA.

Accepted for publication 8 January 1985.

Correspondence to Dr D J Walker, Department of Rheumatology, Freeman Hospital, Freeman Road, High Heaton, Newcastle upon Tyne NE7 7DN.

*Deceased.
Studies have usually been performed on populations ascertained from hospital records. Such patients are likely to be the more severe RA sufferers. A recent population-based study failed to show a DR4 association, except with the more severe, erosive, seropositive disease. ${ }^{7}$ It is possible therefore that DR4 or a linked gene may determine only the severity of an existing disease and not the primary predisposition. The observation that clinically milder disease occurs in HLA-DR2 patients when compared with HLA-DR4 patients supports the hypothesis that DR antigens may influence disease severity. ${ }^{8}$

If genetic factors have a major role in the aetiology of RA, then they should be most easily shown in multicase families. RA as seen in hospitals shows a wide range of severity, and it is likely that the spectrum continues to milder forms that are not referred to hospital. In order to include these individuals it is necessary to either perform a population-based study or to extend the pedigrees to include milder forms of the disease. The prevalence 
of RA in the general population means that large numbers of people need to be seen to include only a few RA sufferers. Population studies are therefore time-consuming and very expensive. We have studied extended multigeneration families in the expectation that we would see the full spectrum of RA from the severe hospital-based index cases to the very mild forms of the disease. Our purpose was to investigate any association between HLA antigens and the presence and severity of RA.

\section{Patients and methods}

\section{PATIENTS}

Families with two or more living members affected by RA were selected. The probands were identified from patients attending the Department of Rheumatology in Newcastle upon Tyne. Attempts were made to interview and study all living family members over the age of 15 years. $83 \%$ of first and second degree relatives and $75 \%$ of all possible family members were seen. One family included sixth degree relatives. All family members were assessed for symptoms and signs of joint disease. The diagnosis of RA was established by the ARA criteria. A history of antirheumatic therapy was taken, with particular reference to the use of gold and penicillamine (second-line drugs).

A total of 219 individuals from 13 families was studied. Thirty-nine individuals suffered from classical or definite RA. A further group of 19 was defined. Six of these had a history of an inflammatory, disabling polyarthritis in a peripheral, symmetrical distribution lasting from several months to 10 years before resolving completely. Most frequently this disease occurred in women of child-bearing age. The remaining 13 individuals fulfilled the ARA criteria for probable RA. No other cause was present for their mild, inflammatory arthropathy. It is of interest that only two of these had been referred to hospital for arthritic problems.

\section{METHODS}

Blood was taken from all family members for HLA typing and measurement of rheumatoid factor. Rheumatoid factor was measured by the RA hyaluronic acid test which detects IgM rheumatoid factor. A titre of $1 / 40$ was taken as positive. HLA typing was performed by a standard lymphocytotoxic assay from $A, B, C$, and DR isoantigens as described by Dewar. ${ }^{10}$ The lymphocyte separation was performed on heparinised blood by the dextran sedimentation technique with added carbonyl iron and centrifugation over a density gradient (Ficoll-Isopaque sp gr 1.078). The $T$ and $B$ cell populations were separated by rosetting the $T$ cells with neuraminidase-treated sheep red blood cells plus added polybrene and $\stackrel{\text {. }}{\text {. }}$ centrifuging over Ficoll-Isopaque. The lymphocyto- $\overrightarrow{\vec{F}}$ toxic technique used was a modification of the method suggested by Mitall et al. ${ }^{12}$ A test was made $\frac{C}{O}$ for DR specificities one to ten.

The severity of the RA was assessed by clinical $\frac{\sqrt{\sigma}}{\vec{D}}$ and radiological indexes. A score for clinical severity was achieved by scoring joints for pain, swelling, ores deformity on a five-point scale (0-normal, 1-pain, $\overrightarrow{0}$ 2-swelling, 3-pain and swelling, 4-deformed). One score was taken for each of the following pairs of groups of joints: proximal interphalangeal (PIP), metacarpophalangeal (MCP), wrist, elbow, $\stackrel{0}{\circ}$

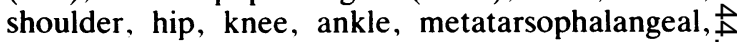
and cervical spine, and one score for the worst otheroo affected joint (maximum possible $=44$ ). We have $\stackrel{G}{\oplus}$ called the total the spread/severity index (SS index). $X$-rays were performed on those with symptoms or signs of inflammatory arthropathy. $X$-ray grading $\vec{\longrightarrow}$ was performed on hand $x$-rays by comparison with standard radiographs on a $0-5$ scale. ${ }^{13}$ The score wasc taken for MCPs, PIPs, and wrist joints, and the total score was divided by seven for an overall grade $\vec{\infty}$ (maximum grade $=8$ ). Statistical analysis for associa- -0 tion was carried out by the $\chi^{2}$ test. Analysis of severity indicators was by the Mann-Whitney U test.

\section{Results}

The family population in total showed a high $\stackrel{2}{\rightarrow}$ frequency of HLA-DR4. This antigen was present in $\frac{3}{3}$ 149 individuals out of $219(68 \%)$. A random sample of the local population showed the presence of HLA-DR4 in 70 out of $200(35 \%)$. HLA-DR2 showed a low frequency, being present in 21 out of the 219 family population $(10 \%)$. In the randomo sample DR2 occurred in 64 out of the $200(32 \%)$

HLA-DR ASSOCIATION WITH RA Thirty-five of 39 members $(90 \%)$ with classical oro definite RA possessed HLA-DR4 (Table 1). This is $\triangle$ a significant association when compared with the non-RA family members $(p<0 \cdot 01)$. Twelve of these RA sufferers were homozygous for HLA-DR4. There were 16 non-RA homozygotes for HLA-DR4 0 (eight female and eight male; average age 54 years,,$\omega$ range 20-77 years). Homozygous HLA-DR4 was just significantly more associated with RA thano heterozygous HLA-DR4 $(p=0 \cdot 05)$. HLA-DR2, though having a very low frequency within the? families, was not in negative association with RAD (Table 1). Of the 19 patients with probable or previous RA $13(68 \%)$ possessed HLA-DR4, and of these two were homozygous for HLA-DR4. $\stackrel{\mathbb{Q}}{\Omega}$ These figures do not differ significantly from the non-RA family members. 
Table 1 HLA-DR association with $R A$

\begin{tabular}{lcccc}
\hline & Classical and definite $R A$ & Probable and previous RA & Non-RA & $\begin{array}{c}\text { Random local } \\
\text { population }\end{array}$ \\
\hline Total & 39 & 19 & 161 & 200 \\
HLA-DR4 positive & $35(90 \%)^{*}$ & $13(68 \%)$ & $101(63 \%)$ & $70(35 \%)$ \\
HLA-DR4 homozygous & 12 & 2 & 16 & $46(29 \%)$ \\
Haplotype present & $32(83 \%)^{*}$ & $7(37 \%)$ & $17(11 \%)$ & $64(32 \%)$ \\
HLA-DR2 positive & $2(5 \%)^{\dagger}$ & $2(11 \%)$ & & \\
\hline
\end{tabular}

${ }^{*} v$ non-RA; $\mathrm{p}<0.01$. $+v$ non-RA; $\mathrm{p}$ not significant.

SER O POS I T I V I TY

$72 \%$ of the classical and definite RA sufferers were seropositive. The incidence of HLA-DR4 was similar in the seropositive and seronegative groups (Table 2). Non-RA seropositivity occurred in two individuals, one of whom possessed HLA-DR4. These figures suggest that HLA-DR4 was not associated with IgM seropositivity in itself but with 'rheumatoid disease'.

The haplotypes found in the affected family members are listed in Tables 3 and 4 . Table 3 shows that in all the families there was evidence of haplotype sharing between affected members. In family 01 there was no haplotype sharing in members with classical or definite RA. In families 08 and 12 two haplotypes were shared by members with classical or definite RA. In families 01 and 08 the most commonly shared haplotype ('RA' haplotype) was identified by inclusion of affected members with probable or previous RA. Both haplotypes are shown for family 12 . Table 4 shows the HLA A, B, $C$, and DR antigens of these shared haplotypes.

Thirty-two of the 39 classical or definite RA sufferers possessed the haplotype for their family (Table 1). Of the probable or previous RA sufferers seven had inherited the haplotype for their family. This was not different from the non-RA family members. Again, the haplotype was shown to be associated with the disease rather than IgM seropositivity (Table 2 ).

Fig. 1 shows the age of onset and the severity indicators and their relationship to HLA-DR4

Table 2 HLA-DR4 and haplotype associations with seropositivity

\begin{tabular}{llrrr}
\hline & & Total & HLA-DR4 positive (\%) & Haplotype positive (\%) \\
\hline Classical and definite RA & Seropositive & 28 & $26(93 \%)$ & $24(86 \%)$ \\
Non-RA & Seronegative & 11 & $9(82 \%)$ & $8(73 \%)$ \\
& Seropositive & 2 & $1(50 \%)$ & 0 \\
& Seronegative & 159 & $100(63 \%)$ & $46(29 \%)$ \\
\hline
\end{tabular}

Table 3 Distribution of lettered haplotypes in affected individuals in each family

\begin{tabular}{|c|c|c|c|c|c|c|c|c|c|c|c|c|}
\hline \multirow[t]{3}{*}{ Family } & \multicolumn{9}{|c|}{ Classical and definite $R A$} & \multicolumn{3}{|c|}{ Probable and previous $R A$} \\
\hline & \multicolumn{12}{|c|}{ Individual } \\
\hline & 1 & 2 & 3 & 4 & 5 & 6 & 7 & 8 & 9 & 1 & 2 & 3 \\
\hline 01 & $\mathbf{a b}$ & cd & ef & & & & & & & ag & & \\
\hline 02 & $a b$ & ac & & & & & & & & de & & \\
\hline 03 & ab & ac & ad & be & ad & af & gh & ig & ai & gh & kl & am \\
\hline 04 & $\mathbf{a b}$ & ac & & & & & & & & cd & ae & \\
\hline 05 & $\mathbf{a b}$ & $\mathrm{ac}$ & & & & & & & & ad & cd & \\
\hline 06 & ab & ac & & & & & & & & bd & & \\
\hline 07 & $\mathbf{a b}$ & ac & ad & & & & & & & de & $\mathbf{a b}$ & \\
\hline 08 & $\mathbf{a b}$ & $\mathbf{a b}$ & & & & & & & & $\mathrm{cd}$ & ac & \\
\hline 09 & $a b$ & $\mathrm{ac}$ & & & & & & & & $\mathrm{cd}$ & & \\
\hline 10 & ab & ac & ad & ef & & & & & & ad & gh & \\
\hline 11 & $a b$ & ac & ac & & & & & & & cd & & \\
\hline 12 & ab & $a b$ & & & & & & & & cd & & \\
\hline 13 & $a b$ & $\mathrm{ac}$ & & & & & & & & & & \\
\hline
\end{tabular}


Table 4 Haplotype a (' $R A$ ' haplotype) from Table 3 identified by $A, B, C$, and $D R$ type

\begin{tabular}{|c|c|c|c|c|c|}
\hline \multirow[t]{2}{*}{ Family } & & \multicolumn{4}{|c|}{ HLA locus } \\
\hline & & $A$ & $B$ & $C$ & $D R$ \\
\hline & 29 & 45 & 6 & $4^{*}$ \\
\hline \multicolumn{2}{|l|}{$\begin{array}{l}01 \\
02\end{array}$} & 1 & 8 & - & $4^{* *}$ \\
\hline \multicolumn{2}{|l|}{03} & 31 & 7 & - & $4^{* * *}$ \\
\hline \multicolumn{2}{|l|}{04} & 31 & 62 & 3 & 4 \\
\hline \multicolumn{2}{|l|}{05} & 2 & 44 & 5 & 4 \\
\hline \multicolumn{2}{|l|}{06} & 32 & 44 & 5 & 4 \\
\hline \multicolumn{2}{|l|}{07} & 1 & 8 & - & $4^{* *}$ \\
\hline \multicolumn{2}{|l|}{08} & 1 & 44 & 5 & 4 \\
\hline \multicolumn{2}{|l|}{09} & 3 & 5 & - & 6 \\
\hline \multicolumn{2}{|l|}{10} & 31 & 7 & - & $4^{* * *}$ \\
\hline 11 & & 29 & 45 & 6 & $4^{*}$ \\
\hline \multirow{2}{*}{$12 \dagger$} & $\{(a)$ & 2 & 40 & 3 & $4\}$ \\
\hline & $\{$ (b) 2 & 29 & 44 & - & $-\}$ \\
\hline \multicolumn{2}{|l|}{13} & 2 & 44 & - & 1 \\
\hline
\end{tabular}

${ }^{*}$ Indicate shared haplotypes.

†Haplotypes $a$ and $b$ are shown, as it was impossible to identify the 'RA' haplotype in this family.

status. The small number of DR4 negative individuals prevents valid comparison with DR4 positive individuals. We were, however, able to compare RA sufferers for homozygosity and heterozygosity fot: DR4. No difference was found for classical and definite RA sufferers for age of onset or severityo This relationship was not altered by the inclusion of the probable and previous group. Seven of the $1 \overline{2 n}$ homozygous, 11 of the 23 heterozygous, and none of the four non-DR4 patients had been treated with second-line drugs. There was no difference in the duration of the disease in the three groups? Homozygosity for DR4 did not therefore lead to earlier onset or more severe disease by comparisom with heterozygous RA.

Fig. 2 shows the relationship between severity indicators and the inheritance of the 'RA' haplotypes for their family. The age of onset was not differenf for the two groups. The severity index showed $\overrightarrow{c_{0}}$ trend towards the haplotype possessors having moreo severe disease for the classical definite RA group? When the probable and previous group was in $>$ cluded this difference was significant at $\mathrm{p}<0.01$ fow both SS index and $x$-ray grade.

Seventeen of the 32 classical and definite RA sufferers inheriting the haplotype had been treate 60 with second-line drugs compared with one of the seven without the haplotype. There was no differ-

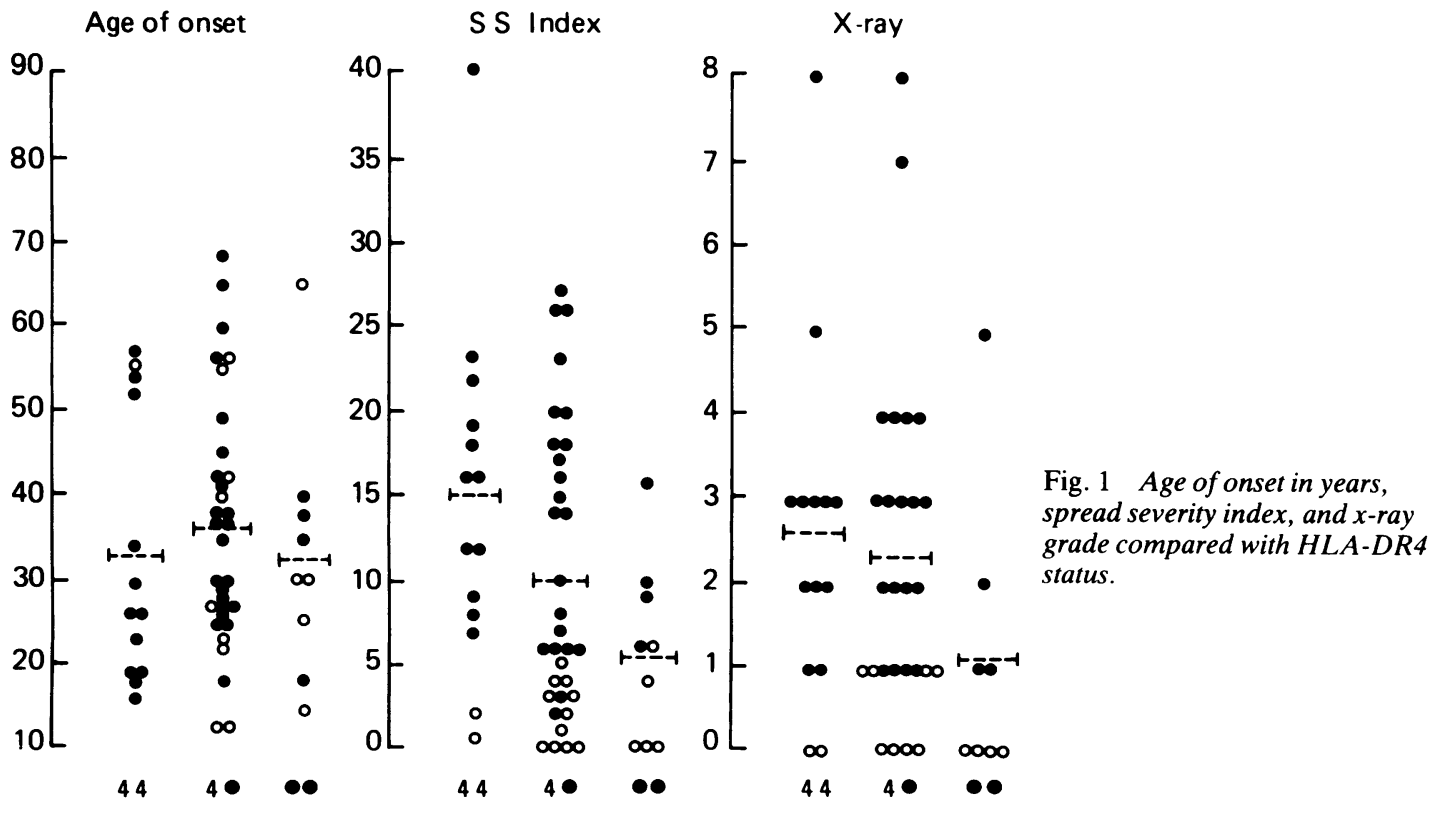

- Classical or definite R A

C Probable or previous R A Homozygous HLA DR4

- Heterozygous HLA DR4

- No HLA DR4 

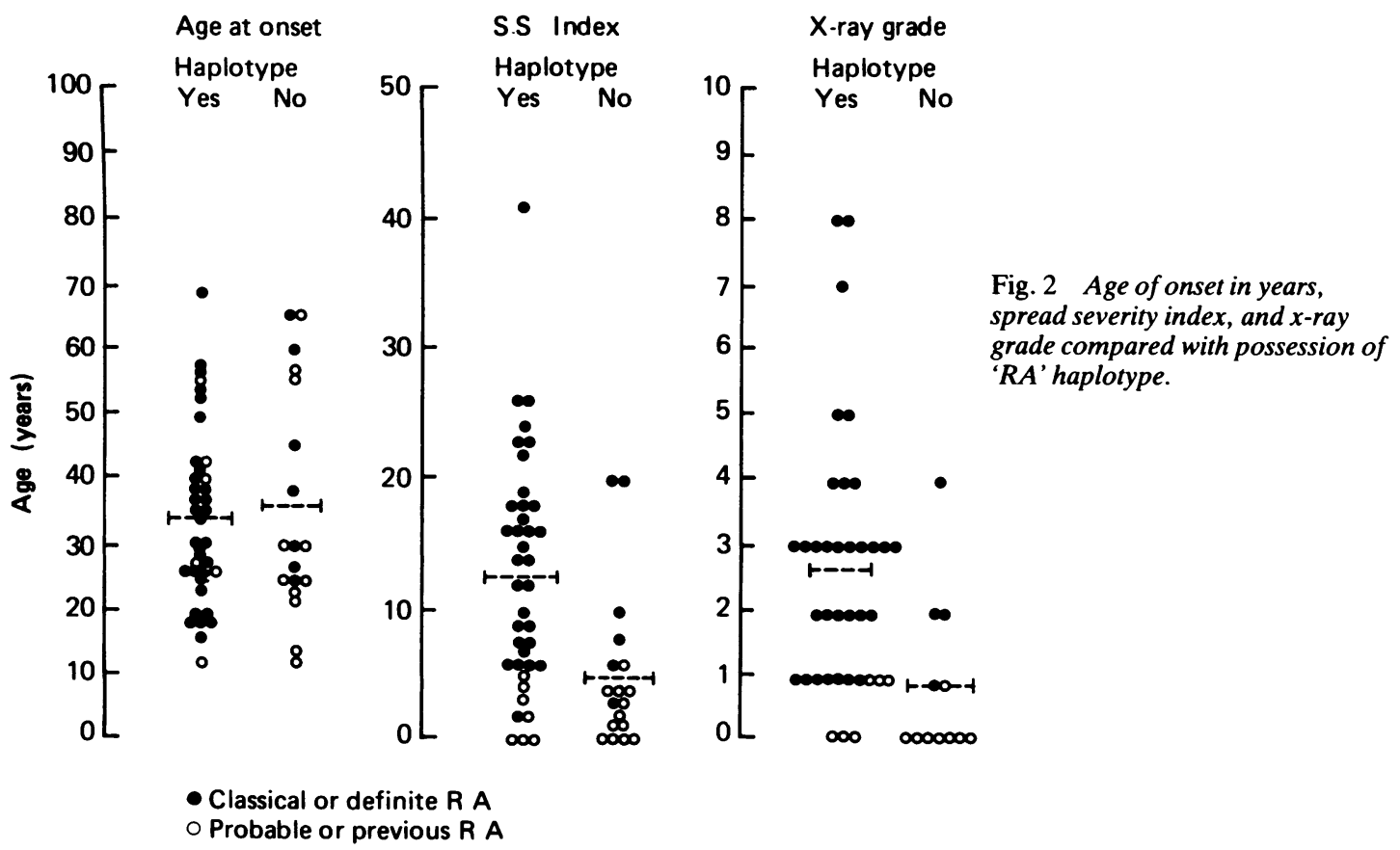

ence in duration of disease between the two groups. These results suggest that the inheritance of the 'RA' haplotype did not lead to earlier onset but may lead to more severe disease.

In order to separate the effect of the haplotype and HLA-DR4 those possessing the haplotype but not HLA-DR4 should be compared with those possessing DR4 but not the haplotype. There were three individuals with classical or definite RA in the former group and six in the latter. With these small numbers statistical testing was not appropriate.

\section{Discussion}

Our results confirm the association of classical and definite RA with HLA-DR4 and show that the association remains true within families. The high frequency of HLA-DR4 within the unaffected family members compared with the local control population is probably secondary to this association. Conversely the very low frequency of HLA-DR2 may suggest that RA is negatively associated with HLA-DR2. The failure of the RA group to show this negative association significantly may be due to the small number of the HLA-DR2 individuals in the families.

The role of the MHC in the pathogenesis of RA

remains ill defined. HLA-DR4 or a linked gene may act as either a susceptibility or severity gene for RA. As there is no pathognomic test for RA, diagnosis depends on the summation of clinical, laboratory, and radiological features; the disease being graded from 'possible' to 'classical', depending on the number of features present. This grading reflects not only the confidence of diagnosis but also the severity of the disease. Individuals possessing a severity marker are more likely to achieve the necessary criteria for classical or definite diagnosis. Either the susceptibility or the severity hypothesis would therefore explain the observed association of HLA-DR4 with classical or definite RA.

Variable age of onset, complex and uncertain diagnostic criteria, and pedigree evidence of variable penetrance make classic linkage analysis difficult and tend to conceal true linkage. Conversely the possible effect of the MHC on disease severity which influences diagnosis could indicate linkage where none exists. Linkage analysis data therefore are difficult to interpret.

A disease susceptibility gene should be associated with the full spectrum of the disease, whereas a disease severity gene will only be associated with the more severe forms. The groups of probable and previous RA may represent the mild end of the 
spectrum of RA, though some diagnostic uncertainty must exist. We have been unable to show any HLA association with this group compared with unaffected family members. This observation supports the severity hypothesis. HLA-DR4 has in the past been linked with more severe disease ${ }^{14}$ and a stronger association has been shown with extraarticular disease such as Felty's syndrome. ${ }^{15}$ If HLA-DR4 is a severity marker, then those homozygous for HLA-DR4 may suffer from more severe disease. This was found by Brackertz and Wernet ${ }^{16}$ who investigated unrelated individuals, but it is not supported by our results.

If the HLA association of RA is with a gene in the MHC other than DR4, then analysis of the complete haplotypes should show stronger association. We therefore selected the haplotypes which seemed most commonly inherited with the RA in individual families. In this way we were able to select haplotypes which overall are at least as strongly associated with RA as HLA-DR4.

When an analysis for the severity of the disease was carried out, an association between haplotype inheritance and more severe disease was shown when the probable and previous group were included. This result therefore depends on the probable and previous group being mild forms of the same disease process. These 'mild forms' of RA had not been referred to hospital and were only discovered by studying extended pedigrees.

The observation that properdin factor phenotypes are associated with RA and that this association can be independent of linkage disequilibrium with HLA-DR $4^{6}$ is evidence that there are genes in the MHC which are separate from the DR locus and govern susceptibility or severity. A recent report of MHC associations with radiological severity shows an association between possession of HLA-Dw4 and HLA-DR4 and more severe erosions. ${ }^{17}$ A haplotype containing both HLA-Dw4 and HLA-DR4 may therefore be more associated with severity than HLA-DR4 alone. This again would suggest that it is not DR4 in itself but a linked gene which is important. The presence of classical and definite RA sufferers who do not possess HLA-DR4 but inherit the haplotype for the family is some support for this theory.

Haplotypes and parts of haplotypes have been associated with RA in previous family studies. ${ }^{18-20}$ No individual haplotype, as specified by A, B, C, and D or DR loci, has shown a consistent association throughout the reported families. Our findings support the lack of individual haplotype association.

Population surveys have suggested that only seropositive RA is familial. ${ }^{21}$ Subsequent reports have suggested that HLA-DR4 is associated with only seropositive disease, ${ }^{22}$ and one report has shown association of HLA-DR4 with high titr产 seropositivity. ${ }^{23}$ These findings have not been con firmed by Panayi et al, ${ }^{24}$ and HLA-DR4 is not foung to be associated with non-RA seropositivity. ${ }^{25}$ Ou $\overline{\bar{w}}$. results support the view that HLA-DR4 is associ ated with rheumatoid disease rather than $\operatorname{Ig}\left(\frac{6}{6}\right.$ seropositivity.

This work was supported by grants from the Arthritis and Rheumatism Council and the Newcastle DHA Research Commiơ tee. We wish to thank Miss K Lumley for her secretarial assistance्य and Dr J Burn for his advice.

\section{References}

1 Lawrence J S. Rheumatoid arthritis. London: Heinemann 1977: 156-271.

2 McMichael A J, Sasazyki T, McDevitt H O, Payne R D Increased frequency of HLA-Cw3 and HLA-Dw4 in rheumatoid arthritis. Arthritis Rheum 1977; 20: 1037-42.

3 Stastny P. Association of the B cell alloantigen DRw4 wit rheumatoid arthritis. N Engl J Med 1978; 298: 869-71.

4 Panayi G S, Wooley P, Batchelor J R. Genetic basis @ rheumatoid disease: HLA antigens, discase, manifestation $\overrightarrow{0}$ and toxic reactions to drugs. $\mathrm{Br}$ Med J 1978; ii: 1326-8.

5 O'Neill G J, Nerl C W, Kay P H, Christiansen F T, McCluskefy J, Dawkins $\mathbf{R}$ L. Complement $\mathrm{C4}$ is a marker for adul rheumatoid arthritis. Lancet 1982; ii: 214.

6 Dyer P, Grennan D M, Walton K, et al. HLA and properdin factor B (Bf) in rheumatoid arthritis. Ann Rheum Dis 1984; 439 118.

7 De Jongh B M, Van Romunde L K J, Valkenburg H A, D융 Lange $G$ G, Vanrood J J. Epidemiological study of HLA and GM in rheumatoid arthritis and related symptoms in an ope Dutch population. Ann Rheum Dis 1984; 43: 613-9.

8 Griffin A J, Wooley P, Panayi G S, Batchelor J R. HLA DB antigens and disease expression in rheumatoid arthritis. $A$ 年 Rheum Dis 1984; 43: 218-21.

9 Ropes M W, Bennett G A, Cobb S, Jacox R, Jessar R A Revision of diagnostic criteria for rheumatoid arthritis. Buף Rheum Dis 1958; 9: 175-6.

10 Dewar P J. HLA antigens. Clin Rheum Dis 1983; 9: 103-5?

11 Gelsthorpe K, Doughty $\mathrm{R}$ W. B lymphocyte preparation ang typing in less than four hours. Tissue Antigens 1977; 10: $236 \frac{3}{3}$

12 Mittal K K, Mickey M R, Singal D P, Terasaki P I. Serotyping for homotransplantation. XVIII. Refinement of microdrople lymphocyte cytotoxicity test. Transplantation 1968; 6: 913-27

13 Larsen A, Dale K. Standardised radiological evaluation $\mathbf{\alpha}$ rheumatoid arthritis. In: Dumonde D C, Steward M W, eds. Laboratory tests in rheumatic disease. Lancaster: MTP Pres 1979: $59-70$.

14 Roitt I M, Corbett M, Festenstein H, et al. HLA-DRw4 anf prognosis in rheumatoid arthritis. Lancet 1978; i: 990.

15 Dinant H J, Hissink Muller W, Van den Berg-Loonen E M Nijenhuis L E, Engelfriet C P. HLA-DRw4 in Felty's syn drome. Arthritis Rheum 1980; 23: 1336.

16 Brackertz D, Wernet P. Genetic analysis of rheumatoif arthritis: population and family studies. Arthritis Rheum 1980? 23: 656 .

17 Young A, Jaraquemada D, Awad J, et al. Association of HLA DR4/Dw4 and DR2/Dw2 with radiological changes in prospective study of patients with rheumatoid arthritis. Arthrit Rheum 1984; 27: 20-5.

18 Khan M A, Kushner I, Braun W E, Dejelo C L, Ballou S P. Clinical and HLA studies in multiple case families wit rheumatoid arthritis. Tissue Antigens 1981; 18: 136-8 
19 Ström H, Möller E. HLA and rheumatoid arthritis. A study of 5 families. Tissue Antigens 1981; 18: 92-100.

20 Nunez G, Moore S, Ball B V. Hurd E, Khan M A. Stastny P. The inheritance of HLA haplotypes in families with adult rheumatoid arthritis. Arthritis Rheum 1980; 23: 726.

21 Lawrence J S. Ball J. Genetic studies in rheumatoid arthritis. Ann Rheum Dis 1958; 17: 160-8.

22 Dobloug J H, Førre $\emptyset$, Kåss E. Thorsby E. HLA antigens and rheumatoid arthritis (association between HLA-DRw4 positiv- ity and IgM rheumatoid factor production). Arthritis Rheum 1980; 23: 309-13.

23 Stastny P. Rheumatoid arthritis. In: Terasaki P, ed. Histocompatability testing. Los Angeles: UCLA Press. 1980; V: 681-6.

24 Panayi G S. Wooley P. Batchelor J R. HLA-DR4 and rheumatoid arthritis. Lancet 1979; i: 730 .

25 Engleman E G. Sponzilli E E. Batey M E, Ramcharan S, McDevitt H O. Mixed lymphocyte reaction in healthy women with rheumatoid factor. Arthritis Rheum 1978; 21: 690-3. 